\title{
TRANSFORMS VANISHING AT INFINITY \\ IN A CERTAIN DIRECTION \\ AND SEMI-IDEMPOTENT MEASURES
}

\section{LOUIS PIGNO}

Let $G$ be a compact abelian group with character group $\Gamma$. Throughout the paper, we shall assume there is a non-trivial group homomorphism

$$
\varphi: \Gamma \rightarrow \mathrm{R}
$$

where $\mathbf{R}$ is the additive group of reals. If $\varphi$ is an isomorphism, then the semigroup $\mathscr{P}$ is the set $\{\gamma \in \Gamma: \varphi(\gamma) \geqq 0\}$. For convenience, assume $\Gamma$ is countable.

Let $M(G)$ be the usual convolution algebra of finite complex-valued Borel measures on $G$. The Fourier-Stieltjes transform of the measure $\mu \in M(G)$ is the function $\hat{\mu}$ defined on $\Gamma$ by

$$
\hat{\mu}(\gamma)=\int_{G} \gamma(-x) d \mu(x) .
$$

We will also use ${ }^{\wedge}$ to denote the Gelfand transform. Let $M_{0}(G)$ be the ideal of measures $\mu \in M(G)$ such that $\hat{\mu} \in C_{0}(\Gamma)$.

We designate by $M_{\varphi}(G)$ the set of those $\mu \in M(G)$ such that $\hat{\mu}$ vanishes at infinity in the direction of $\varphi$. By this is meant $\left\{\gamma_{n}\right\}_{1}^{\infty} \subset \Gamma$ with $\varphi\left(\gamma_{n}\right)$ $\rightarrow \infty \Rightarrow \hat{\mu}\left(\gamma_{n}\right) \rightarrow 0$. Here, $\varphi\left(\gamma_{n}\right) \rightarrow \infty$ in the usual topology of $\mathbf{R}$.

It is easy to check that $M_{\varphi}(G)$ is a closed ideal of $M(G)$ such that if $\tau \in M_{\varphi}(G)$ and $\xi \ll \tau$ then $\xi \in M_{\varphi}(G)$. Thus

$$
M(G)=M_{\varphi}(G) \oplus M_{\varphi}^{\perp}(G)
$$

where

$$
M_{\varphi}^{\perp}(G)=\left\{\varrho \in M(G): \varrho \perp \tau \text { for each } \tau \in M_{\varphi}(G)\right\} .
$$

Let $\delta_{0}$ be the identity measure in $M(G)$ and for any set of non-zero integers $\left\{N_{1}, \ldots, N_{m}\right\}$ put $\delta_{i}=N_{i} \delta_{0}, i=1,2, \ldots, m$. We state our first result.

THeOREM 1. Let $\mu \in M(G)$ with $\mu * \prod_{i=1}^{m}\left(\mu-\delta_{i}\right) \in M_{\varphi}(G)$. Then $\mu=\mu_{0}+\mu_{\perp}$ where $\mu_{0} \in M_{\varphi}(G), \mu_{\perp} \in M_{\varphi}^{\perp}(G)$ and $\hat{\mu}_{\perp}(\Gamma) \subset Z$.

Received July 27, 1976; in revised form September 19, 1976. 
Proof. We adapt the method of [5]: Suppose $\mu \in M(G)$ and

$$
\mu * \prod_{i=1}^{m}\left(\mu-\delta_{i}\right) \in M_{\varphi}(G) \text {. }
$$

Since $M_{\varphi}(G)$ is an ideal (1) gives:

$$
\mu_{\perp} * \prod_{i=1}^{m}\left(\mu_{\perp}-\delta_{i}\right) \in M_{\varphi}(G) .
$$

Let $S$ be the structure semi-group for $M(G)$ and consider the image of $M(G)$ in $M(S)$ in the usual way; see [8]. For $\xi \in M(G)$ the image of $\xi$ is denoted by $(\xi)_{s}$. Then (2) becomes:

$$
\left(\mu_{\perp}\right)_{s} * \prod_{i=1}^{m}\left(\mu_{\perp}-\delta_{i}\right)_{s} \in M_{\varphi}(S)
$$

where $M_{\varphi}(S)$ is the image of $M_{\varphi}(G)$.

We shall assume

$$
\left(\mu_{\perp}\right)_{s} \neq 0 \text {. }
$$

Let $\hat{\boldsymbol{S}}$ denote the semi-characters of $\boldsymbol{S}$ and $\bar{\Gamma}$ the closure of $\Gamma$ in $\hat{\boldsymbol{S}}$. Recall that $\hat{\boldsymbol{S}}$ is the maximal ideal space of $M(G)$. Now (4) implies the existence of an infinite set $\left\{\gamma_{n}\right\}_{1}^{\infty} \subset \Gamma$ and an $\varepsilon>0$ such that $\left|\hat{\mu}_{\perp}\left(\gamma_{n}\right)\right| \geqq \varepsilon$ and $\varphi\left(\gamma_{n}\right) \rightarrow \infty$.

Thus $\left\{\gamma_{n}\right\}_{1}^{\infty}$ has a cluster point $\beta_{0} \in \bar{\Gamma} \backslash \Gamma$. Since conjugation is continuous and multiplication of semi-characters separately continuous we may infer that $\left|\beta_{0}\right|^{2} \in \bar{\Gamma} \backslash \Gamma$.

Put $\boldsymbol{T}\left(\beta_{0}\right)=\left\{s \in S: \beta_{0}(s)=0\right\}$. Then define

$$
\left(\mu_{\perp}\right)_{s}=\mu_{1}+\mu_{2}
$$

where

$$
\mu_{1}=\left.\left(\mu_{\perp}\right)_{s}\right|_{T\left(\beta_{0}\right)} \quad \text { and } \quad \mu_{2}=\left.\left(\mu_{\perp}\right)_{s}\right|_{S \backslash T\left(\beta_{0}\right)} .
$$

Notice $\mu_{2} \neq 0$ by (4).

Let $\tau \in M_{\varphi}(S)$ and consider

$$
|\tau|^{\hat{(}}\left(\left|\beta_{0}\right|^{2}\right)=\int_{\boldsymbol{S} \backslash \boldsymbol{T}\left(\beta_{0}\right)}\left|\beta_{0}\right|^{2}(s) d|\tau|(s) .
$$

Now for fixed $k$ we have $\lim _{j} \varphi\left(\gamma_{j}-\gamma_{k}\right)=\infty$, so since the Gelfand transform is continuous on $\hat{S}$ and multiplication of semi-characters separately continuous we may conclude that $|\tau|^{\hat{\prime}}\left(\left|\beta_{0}\right|^{2}\right)=0$. Thus, we gather from (5) that $M_{\varphi}(S)$ is carried by $T\left(\beta_{0}\right)$. Recall for any $\omega_{1}, \omega_{2} \in M(S)$ that

$$
\text { carrier }\left(\omega_{1} * \omega_{2}\right) \subset\left(\text { carrier } \omega_{1}\right)\left(\text { carrier } \omega_{2}\right) .
$$


Inasmuch as $\boldsymbol{T}\left(\beta_{0}\right)$ is an ideal and $\boldsymbol{S} \backslash \boldsymbol{T}\left(\beta_{0}\right)$ is a semigroup we obtain via (6) that for any $\omega \in M(S)$ and $\tau \in M_{\varphi}(S)$ the condition:

$$
\mu_{2} * \prod_{i=1}^{m}\left(\mu_{2}-\left(\delta_{i}\right)_{s}\right) \perp \tau+\omega * \mu_{1} .
$$

We gather from (3) and (7) that

$$
\mu_{2} * \prod_{i=1}^{m}\left(\mu_{2}-\left(\delta_{i}\right)_{s}\right)=0 .
$$

Pulling back, we have

$$
\mu_{\perp}=\varrho_{1}+\varrho_{2}, \quad \varrho_{1} \perp \varrho_{2}
$$

where $\left(\varrho_{i}\right)_{s}=\mu_{i}, i=1,2$. As a consequence of (8)

$$
\varrho_{2} * \prod_{i=1}^{m}\left(\varrho_{2}-\delta_{i}\right)=0, \quad\left(\varrho_{2} \neq 0\right) .
$$

Since $\varrho_{i} \in M_{\varphi}^{\perp}(G)(i=1,2)$ we see from $(10)$ that $\left\|\mu-\varrho_{2}\right\| \leqq\|\mu\|-1$. So if $\varrho_{1} \neq 0$ we apply this finite descent argument to $\mu-\varrho_{2}$ and therefore conclude that

$$
\hat{\mu}_{\perp}(\Gamma) \subset \mathbf{Z} \text {. }
$$

This completes the proof.

Theorem 1 has an application to semi-idempotent measures which we now give. A subset $E$ of $\Gamma$ is said to be a Sidon set if $f \in L^{\infty}(G)$ with $\operatorname{supp} \hat{f}$ $\subset E \Rightarrow \Sigma|\hat{f}(\gamma)|<\infty$. For any subset $A$ of $\Gamma$ put

$$
\boldsymbol{F}(A)=\{\mu \in M(G): \hat{\mu} \text { is integer-valued on } A\}
$$

and

$$
I(A)=\left\{\mu^{\circ} \in M(G): \hat{\mu}=0 \text { or } 1 \text { on } A\right\} .
$$

Assume $\varphi$ is a non-trivial isomorphism of $\Gamma$ into $\mathrm{R}$. The following result is an analogue of a result announced by I. Kessler [1]. See also Y. Meyer [3, pp. 206-211].

THEOREM 2. Let $E$ be a Sidon subset of $\Gamma$. Suppose $\mu \in \boldsymbol{F}(\Gamma \backslash-\mathscr{P} \cup E)$. Then there is a $v \in \boldsymbol{F}(\Gamma)$ such that $\hat{\mu}=\hat{v}$ off $-\mathscr{P} \cup E$. In particular, if $\mu \in$ $\boldsymbol{I}(\Gamma \backslash-\mathscr{P} \cup E)$ then $v \in \boldsymbol{I}(\Gamma)$.

Proof. Suppose $\mu \in \boldsymbol{F}(\Gamma \backslash-\mathscr{P} \cup E)$ and let $N_{i}(i=1,2, \ldots, m)$ be the distinct non-zero integer-values of $\hat{\mu}$ off $-\mathscr{P} \cup E$. It is apparent that 


$$
\operatorname{supp}\left\{\mu * \prod_{i=1}^{m}\left(\mu-\delta_{i}\right)\right\} \subset-\mathscr{P} \cup E .
$$

where $\delta_{i}=N_{i} \delta_{0}$. By Theorem 2 of $[7$, p. 368] we see that (1) gives:

$$
\left\{\mu * \prod_{i=1}^{m}\left(\mu-\delta_{i}\right)\right\} \in C_{0}(\mathscr{P}) .
$$

As a consequence of (2) we gather that

$$
\mu * \prod_{i=1}^{m}\left(\mu-\delta_{i}\right) \in M_{\varphi}(G) .
$$

Now (3) in combination with Theorem 1 permits the conclusion:

$$
\mu_{\perp} * \prod_{i=1}^{m}\left(\mu_{\perp}-\delta_{i}\right)=0
$$

where $\mu_{\perp} \in M_{\varphi}^{\perp}(G)$. Since $\mu_{\perp} \in \boldsymbol{F}(\Gamma)$ it is evident that $\mu_{0} \in \boldsymbol{F}(\Gamma \backslash-\mathscr{P} \cup E)$. Consider

$$
\mathscr{F}=\left\{\gamma \notin-\mathscr{P} \cup E:\left|\hat{\mu}_{0}(\gamma)\right| \geqq 1\right\} .
$$

We claim $\mathscr{F}$ is a finite subset of $\mathscr{P}$. To establish our claim, we shall assume $\mathscr{F}$ is infinite and force a contradiction:

Suppose $\mathscr{F}$ is infinite. Clearly $0 \leqq \varphi(\mathscr{F}) \leqq M$ for some $M \in \mathrm{R}^{+}$since $\mu_{0} \in M_{\varphi}(G)$. Let $r_{0}$ be the largest accumulation point of the set $\varphi(\mathscr{F})$ and let $\gamma_{j} \in \mathscr{F}$ be a sequence of distinct elements such that $\varphi\left(\gamma_{j}\right) \rightarrow r_{0}$. Then without loss of generality,

$$
\bar{\gamma}_{j} \mu_{0} \rightarrow v \quad \text { weak }-*
$$

where $v$ is singular with respect to Haar measure on $G$. As a consequence of $\gamma_{j} \in \mathscr{F},(5)$ gives:

$$
\hat{v}(0) \neq 0 \text {. }
$$

Now by Theorem 1.4 of $[2$, p. 8$]$

$$
\underline{\lim }\left(E-\gamma_{j}\right) \text { is a finite subset of } \Gamma \text {. }
$$

Thus, except for a possible finite set of positive $\gamma$ 's,

$$
\lim _{j} \hat{\mu}\left(\gamma+\gamma_{j}\right)=\hat{v}(\gamma)=0 .
$$

because $\gamma+\gamma_{j}$ eventually does not belong to $\mathscr{F}$. Appeal to Theorem 1 of [7] yields $\hat{v}(0)=0$ and this contradicts (6).

Thus, $\mathscr{F}$ is a finite set so there is a trigonometric polynomial $p$ on $G$ such that $\hat{p}=\hat{\mu}_{0}$ off $-\mathscr{P} \cup E$ and $\hat{p}=0$ on $-\mathscr{P} \cup E$. Well, for the $v$ of our Theorem, take $v=\mu_{\perp}+p$. This concludes the proof. 
The assumption that $\Gamma$ be countable in our paper is of course inessential. The assumption that $\varphi$ is a non-trivial isomorphism in Theorem 2 is equivalent to $\Gamma$ having an archemedian order.

Let $G$ be a non-discrete LCA group. The method of proof of Theorem 1 yields the following theorem.

THEOREM 3. If

$$
\mu * \prod_{i=1}^{m}\left(\mu-\delta_{i}\right) \in M_{0}(G)
$$

then $\mu$ has a decomposition $\mu=\mu_{0}+\mu_{\perp}$ where $\mu_{0} \in M_{0}(G), \mu_{\perp} \in M_{0}^{\perp}(G)$ and $\hat{\mu}_{\perp}(\Gamma) \subset \mathbf{Z}$.

For discrete $\Gamma$ we call $\mathfrak{R} \subset \Gamma$ a weak Rajchman set if supp $\hat{\mu} \subset \mathfrak{R}$ $\Rightarrow \mu \in M_{0}(G)$. For examples of Rajchman sets, the reader is referred to [6]. An easy consequence of Theorem 3 is:

If $\mu \in \boldsymbol{F}(\Gamma \backslash \mathfrak{R})$ then there is $a v \in \boldsymbol{F}(\Gamma)$ such that $\hat{\mu}=\hat{v}$ off $\mathfrak{R}$. In particular, if $\mu \in \boldsymbol{I}(\Gamma \backslash \mathfrak{R})$ then $v \in \boldsymbol{I}(\Gamma)$.

We remark that it is possible to prove a result which encompasses Theorem 1. For $\Gamma$ discrete suppose $\Phi$ is any family of non-trivial homomorphisms from $\Gamma$ into R. We designate by $M_{\Phi}(G)$ the set of those $\mu \in M(G)$ with the following property: $\left\{\gamma_{n}\right\}_{1}^{\infty} \subset \Gamma$ with $\varphi\left(\gamma_{n}\right) \rightarrow \infty$ for all $\varphi \in \Phi \Rightarrow \hat{\mu}\left(\gamma_{n}\right) \rightarrow 0$. The proof of Theorem 1 can be adapted to obtain our final theorem.

TheOREM 4. If $\mu * \prod_{i=1}^{m}\left(\mu-\delta_{i}\right) \in M_{\Phi}(G)$ then $\mu$ has a decomposition $\mu=\mu_{0}$ $+\mu_{\perp}$ where $\mu_{0} \in M_{\Phi}(G), \mu_{\perp} \in M_{\Phi}^{\perp}(G)$ and $\hat{\mu}_{\perp}(\Gamma) \subset \mathbf{Z}$.

The author takes pleasure in thanking Professor Y. Domar of Uppsala University for helpful correspondence. The results of this paper were announced in [4].

\section{REFERENCES}

1. I. Kessler,Semi-idempotent measures on abelian groups, Bull. Amer. Math. Soc. 73 (1967), 258260.

2. J. M. López and K. A. Ross, Sidon Sets, Marcel Dekker, Inc., New York, 1975.

3. Y. Meyer, Algebraic Numbers and Harmonic Analysis, North Holland Publishing Company, Amsterdam, 1973.

4. L. Pigno, Parts of measures and integer-valued transforms, Bull. Amer. Math. Soc. 83 (1977), 279-280.

5. L. Pigno, Interpolation of integer-valued Fourier-Stieltjes transform off Rajchman sets, Kansas State Univ. Technical Report, No. 54, 1976. 
6. L. Pigno, Fourier-Stieltjes transforms which vanish at infinity off certain sets, Glasgow Math., J. (to appear).

7. L. Pigno, A variant of the F. and M. Riesz Theorem, J. London Math. Soc. (2), 9 (1974), 368370.

8. J. L. Taylor, Measure algebras, CBMS Conference Report no. 16, Amer. Math. Soc., Providence R.I., 1973.

\author{
KANSAS STATE UNIVERSITY \\ MANHATTAN \\ KANSAS 66506 \\ U.S.A.
}

